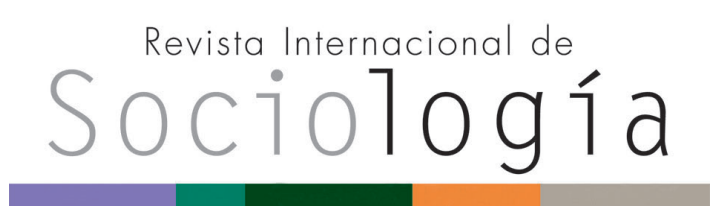

Revista Internacional de Sociología RIS

vol. 75 (3), e068, julio-septiembre, 2017, ISSN-L:0034-9712

doi: http://dx.doi.org/10.3989/ris.2017.75.3.15.117

\section{IDEOLOGÍA Y AVERSIÓN IDEOLÓGICA}

\author{
Vicente Manzano-ArRondo \\ Universidad de Sevilla, España. \\ vmanzano@us.es \\ ORCID iD: http://orcid.org/0000-0003-0456-8480
}

Cómo citar este artículo / Citation: Manzano-Arrondo, V. 2017. "Ideología y aversión ideológica". Revista Internacional de Sociología 75 (3): e068. doi: http://dx.doi.org/10.3989/ ris.2017.75.3.15.117

\section{RESUMEN}

La ideología es un concepto controvertido que genera actitudes muy diversas. Este trabajo propone el constructo "aversión ideológica" para describir una de esas reacciones. La aversión ideológica consiste en la combinación de dos componentes: rechazo de las ideologías y defensa de actuaciones políticas exclusivamente técnicas. Previamente se define la ideología como una forma de ver, preferir y actuar en el mundo, que surge de la relación entre dos dimensiones: encuadres sociales y necesidades. La aversión ideológica responde también a una forma de estar en el mundo, que permite mantener funcionamientos sociales y satisfacer necesidades psicológicas. Este documento ofrece también un instrumento para medir el concepto. El cuestionario consta de diez ítems, cinco para cada uno de los dos componentes de la aversión ideológica. Se ha construido a partir de una muestra on line de $n=650$, y ha sido probada en una muestra cara a cara de $n=340$, mostrando buenas características psicométricas.

\section{Palabras Clave}

Decisión política; Despolitización; Medida de la ideología; Necesidad.

\section{IDEOLOGY AND IDEOLOGICAL AVERSION}

Copyright: @ 2017 CSIC. Este es un artículo de acceso abierto distribuido bajo los términos de la licencia Creative Commons Attribution (CC BY) España 3.0.

Recibido: 01/08/2015. Aceptado: 01/12/2016.

\section{Abstract}

Ideology is a controversial concept which generates very different attitudes. This paper proposes the construct "ideological aversion" to describe one of those reactions. The ideological aversion is a combination of two components: rejection of ideologies, and defense of purely technical policy actions. Previously, ideology is defined as a way of seeing, prefer, and act in the world, which arises from the relationship between two dimensions: social frames and needs. The ideological aversion also responds to a way of being in the world, which keeps social performances and satisfy psychological needs. This document also provides an instrument to measure the concept. The questionnaire consists of ten items, five for each of the two components of the ideological aversion. It has been constructed from an on line sample of $n=650$, and has been tested in a face-to-face sample of $n=340$, showing good psychometric characteristics.

\section{KeYWORDS}

Depoliticization; Measure of ideology; Need; Political Decision. 


\section{INTRODUCCIÓN}

La controversia sobre el contenido y la función de las ideologías acompaña a este concepto desde su nacimiento, habitualmente situado en el cambio del siglo XVIII al XIX (Knight 2006). El constructo se encuentra progresivamente desgastado, denostado $\mathrm{y}$, a la vez, cíclicamente recuperado o dignificado, en una dialéctica que parece acompañarle de forma constante (Orjuela 2007; Ariño 1997; Eagleton 1997). La controversia comienza en su propia definición, sobre la que no existe unanimidad. Y continúa a través de discusiones que se establecen en torno a contemplar el concepto en la práctica, a considerarlo opcional o inevitable, a incluir sus efectos sociales y psicológicos, o a centrar la atención en su relación con otros conceptos pertinentes para entender el comportamiento humano.

Uno de los aspectos más interesantes lo constituyen las actitudes frente al constructo, dentro de las cuales destaca el rechazo a las decisiones mediadas por visiones ideológicas. Algunos autores (ver, por ejemplo, García-Santesmases 2002) describen un hastío generalizado hacia las ideologías, que deriva en la defensa de actuaciones políticas exclusivamente técnicas. Por su parte, Jost et al. (2003) describen el modo en que la "ideología del mercado justo" se presenta a sí misma como un ente a-ideológico que constituye el mejor de los enfoques para tomar decisiones en el ámbito de la política. Este panorama se completa con la exigencia de tomas de decisión únicamente técnicas con que un número creciente de individuos espera encontrar garantías de protección en un mundo competitivo lleno de incertidumbres (Manzano-Arrondo, 2015). A pesar del hastío o del rechazo, se defiende también que las ideologías son entes inevitables (Vázquez 2004; Jost 2006), puesto que el comportamiento humano está mediado por creencias y juicios de valor, que no se encuentran aislados sino organizados precisamente en ideologías. En consecuencia, las decisiones que se toman en el ámbito de la política adquieren contenido y forma desde marcos o referentes ideológicos.

En la justificación de esta controversia participan varios elementos discursivos. Dos de ellos son: (1) el manejo de nociones diferentes e incluso contrapuestas para el concepto; y (2) una comprensión insuficiente sobre el modo en que los marcos ideológicos conectan con la dimensión de las visiones, preferencias y acciones individuales.

Este trabajo se inserta en tal discusión mediante tres aportes. En primer lugar, ofrece una definición de ideología, que pretende ser útil en la controversia, incluyendo en su acotación referencias a la conexión entre el marco ideológico y el ámbito del individuo. En segundo lugar, sugiere el concepto "aversión ideológica" como una postura paradójicamente ideo- lógica que combina dos componentes: la concepción de las ideologías como criaturas perjudiciales y la defensa de actuaciones políticas apoyadas en decisiones exclusivamente mediadas por argumentos técnicos. Por último, provee un instrumento de medida específico para el nuevo constructo.

\section{Concepto de ideología}

En una publicación de 1804, el Conde de Tracy, Antonie Louis Claude Destutt, propone el vocablo ideología para referirse a la ciencia de las ideas, es decir, a la disciplina que aborda el modo en que las personas formulan juicios y generan opiniones. El significado ha variado notablemente, refiriéndose desde hace tiempo no tanto al estudio de las ideas, como a las ideas mismas. Es más, las aproximaciones al concepto coinciden en ir más allá, considerando unidades tan diversas como creencias y hábitos de pensamiento (André 1985), comparaciones sobre qué cosas son importantes frente a otras (Freire 2001), representaciones mentales (Rottenbacher y Schmitz 2012), valores (Garcés 1988), representaciones sociales básicas (Van Dijk 2003), simplificaciones operativas (Brussino et al. 2011), emociones, convicciones y motivaciones (Jost et al. 2003), mitos (Deutsch 2006), legitimizaciones (Kumashiro 2000), significados e identificaciones (Vázquez 2004), sentido de las cosas (Bocock 1995), racionalizaciones (Sibley y Duckitt 2010), posicionamientos políticos (Knight 2006), propuestas de orden social (Jost, Federico y Napier 2009), procesos de cognición social (García-Castro, 2010), reglas y construcciones sociales (Esquivel 2007), etc. La diversidad de acotaciones para el término es tal que Eagleton (1997) considera inviable articular una definición global que pudiera contentar las diferentes aproximaciones, en buena parte incluso incompatibles entre sí.

En un intento de organizar esta diversidad, Ariño (1997) clasifica las propuestas en cuatro categorías: (1) cognitiva crítica, o conjunto de creencias falsas, sesgadas, irracionales, simples o dogmáticas; (2) política crítica, o sistema de ideas que legitiman la dominación; (3) política neutra, o sistema de ideas que legitiman el poder; y (4) semiótica neutra, o sistema de creencias mediante el que se organiza una visión del mundo. En cualquier caso, el objetivo de una definición debe ser, no solo clarificar el concepto para ser compartido, sino procurar un contenido que resulte útil o fructífero en algún sentido. Con este objetivo, propongo la definición que sigue. Si bien se ubica fácilmente en la cuarta categoría de Ariño, los componentes que articula permiten trabajar desde las categorías 2 y 3 , utilizando el concepto como un instrumento de trabajo para la denuncia de procesos ideológicos de opresión o dominación.

La ideología es una forma de ver el mundo (dar cuenta de unos acontecimientos antes que otros 
e interpretarlos de un modo concreto), preferir un mundo (desear o valorar unos estados o funcionamientos antes que otros) y actuar en el mundo (tomar decisiones de acción u omisión), que surge de la relación entre dos niveles: uno macro o contextual y otro micro o psicológico.

En un sentido similar, Jost, Federico y Napier (2009) proponen un sistema de doble sentido para fundamentar la trascendencia y funcionamiento de las ideologías. El camino de arriba hacia abajo (top-down) se nutre de discursos de líderes políticos y de medios de comunicación, entre otras fuentes, construyendo un marco discursivo que suministra herramientas a las personas para orientarse en términos de pensamiento y acción. El camino de abajo hacia arriba (bottom-up) está conformado por rasgos de personalidad, motivaciones, o necesidades, que establecen una vía de encuentro con el marco ideológico. Desde un enfoque parecido, Duckitt y Fisher (2003) plantean un modelo complejo para comprender los posicionamientos ideológicos, considerando, a nivel macro, contextos y visiones sociales, mientras que a nivel micro se localizan actitudes y rasgos de personalidad.

La relación macro-micro alimenta un ciclo de retroalimentación positiva que, no obstante, es permeable a los acontecimientos vitales propios de un mundo dinámico. Los individuos, por ejemplo, pueden vivir experiencias no previstas que les faciliten otorgar credibilidad a marcos distintos o trastocar sus actitudes. En esta línea, el trabajo mencionado de Duckitt y Fisher (2003) recupera hallazgos previos en los que se observa que vivir en contextos sociales de prosperidad disminuye las puntuaciones en la ideología "autoritarismo", mientras que los escenarios amenazantes tienen el efecto contrario.

Por lo general, la relación entre ambos componentes está sujeta a un notable desequilibrio, a favor de la capacidad de influjo de los marcos ideológicos sobre el modo en que los individuos ven, prefieren y actúan, como parte de las dinámicas de poder (ver, por ejemplo, el trabajo de Nafstad et al. 2007). Así, un marco ideológico contiene explicaciones sobre qué es el mundo, cómo funciona y cuál ha de ser el papel del individuo en ello, a través de recursos discursivos (Jost, Federico y Napier 2009), influencia familiar (Block y Block 2006), socialización en las escuelas (Deutsch 2006; Apple 2011), socialización en general (Chatard y Selimbegovic 2008) o mitos legitimadores (Sidanius, Devereux y Pratto 1992; Pratto et al. 1994), entre otras propuestas. No obstante, los individuos no responderían positivamente a estos procesos sociales si no existiera alguna función de nivel micro que justifique las adscripciones o adaptaciones individuales a los marcos ideológicos.

Para describir estas funciones de nivel micro, lo habitual es generar modelos ad hoc de necesidades. Todos ellos fundamentan la conexión entre ambos componentes mediante el mismo esquema: los marcos ideológicos fidelizan a los individuos al mostrarse capaces de saciar alguna necesidad importante y concreta. Así, por ejemplo, en la "creencia en un mundo justo", se acude a la necesidad de las personas de protegerse del temor a las injusticias y establecer proyectos a largo plazo (Maes 1998). La necesidad de protección se consigue asumiendo que cada cual tiene lo que merece y que, por tanto, el buen comportamiento vacuna contra las injusticias (Lerner y Miller 1978). Como corolario, es obvio que las víctimas de las injusticias son culpables de lo que les ha ocurrido; algo han hecho para merecerlo (Hafer y Bègue 2005). De este modo, los estudios muestran relación entre esta creencia y la justificación de violaciones, SIDA, cáncer o accidentes, entre otros sufrimientos (Furnham 2002). En la "justificación del sistema" se organizan hallazgos que explican por qué las personas que sufren las consecuencias de un sistema injusto pueden incluso ser quienes más lo defienden (Jost y Thompson 1999). Jost y colaboradores (Jost et al. 2003; Jost, Banaji y Nosek 2004; Kugler, Jost y Noorbaloochi 2014) acuden principalmente a dos funciones micro para explicar esta aparente incoherencia: (1) la necesidad de evitar la disonancia cognitiva que implicaría aceptar el sistema como injusto y, a la vez, no aplicar esfuerzos en cambiarlo; y (2) la opresión internalizada. Un tercer ejemplo paradigmático lo constituye el "autoritarismo", donde se combina la sumisión a la autoridad, el convencionalismo y el recurso de la violencia para controlar las amenazas al sistema. La adscripción a este marco está asociada a la necesidad de orden (Altemeyer 1999), necesidad de mecanismos de control compensatorio (Kay et al. 2009; Mirisola et al. 2014), así como necesidad de certidumbre y previsibilidad (Kossovska 2003). En la "orientación a la dominancia social” (Sidanius, Devereux y Pratto 1992; Pratto et al. 1994) quienes defienden la supremacía del propio grupo o el disfrute de privilegios, pueden cubrir moralmente su estatus gracias a desviar la responsabilidad hacia el "funcionamiento natural de las cosas", que procura grupos naturalmente dominantes o dominados. En su totalidad, estas explicaciones psicológicas orientadas a la comprensión de los comportamientos individuales, manejan un juego a dos bandas: en términos de Barreiro, Etchezahar y Prado-Gascó (2014) muestran los efectos saludables para los individuos y perjudiciales para la sociedad; en el sentido de que su función de protección individual -aun cortoplacista o parcial- refuerza un funcionamiento social injusto, robusteciendo los desequilibrios de poder.

En este contexto se echa de menos acudir a teorías sobre necesidades que no requieran una construcción ad hoc, sino que hayan sido fundamentadas en un sentido más amplio. Este recurso facilitaría, entre otras posibilidades, estudiar las relaciones macro-micro de diferentes propuestas ideológicas 
utilizando un mismo sistema de análisis. En la actualidad, el modelo más extendido para el estudio de las necesidades humanas es la teoría de la autodeterminación (self-determination theory, SDT) de Deci y Ryan (2000). Según la SDT, existen tres necesidades a la vez universales y psicológicas: "autonomía" -un acto es autónomo si es plenamente respaldado por el individuo que toma la decisión voluntaria de Ilevarlo a cabo-, "competencia" -ser capaz, útil, hábil, tener impacto- y "relación" -interactuar, amar y cuidar, recibir amor y cuidados, sentir cariño y aceptación-. En la SDT no se establece ninguna relación jerárquica entre necesidades. Las personas requieren cubrir las tres, al menos hasta un umbral mínimo, para obtener bienestar. En caso contrario, la consecuencia es la generación de problemas de salud. Es más, satisfacer las tres necesidades hace prescindible cualquier otra (Ryan y Brown 2003). Las ideologías en general permiten saciar con claridad las necesidades de competencia y relación. La competencia se logra gracias a contar con un marco que suministra explicaciones sobre el funcionamiento del mundo y que, por tanto, permite a los individuos elaborar la sensación de que son capaces de entender y moverse por un contexto naturalmente complejo. El recurso de los marcos ideológicos para sostener la necesidad básica de competencia podría explicar la teoría de los efectos limitados (por ejemplo, Lledó et al. 2010), que describe a los individuos como buscadores selectivos de medios de comunicación, escogiendo los que les permiten afianzar sus creencias previas. La tendencia a conectar con iguales, gracias a compartir con ellos la selección del mismo marco ideológico, satisface la necesidad de relación, algo que puede ser también observado desde la teoría de la identidad social (Reicher 2004).

La necesidad de autonomía muestra un papel más complejo en la comprensión de la relación macromicro. Dado que los individuos requieren también saciarla, en una medida no inferior a las otras dos, la adscripción a marcos que no la potencien, debería ir seguida de algún tipo de recurso que solvente el problema. Esta dinámica puede observarse con relativa claridad en el caso del autoritarismo. Así, Duckitt y Fisher (2003) encuentran una relación claramente inversa entre las puntuaciones en las escalas de autoritarismo y de autonomía. A este déficit se le añaden algunas conclusiones del célebre trabajo de Adorno et al. (1950), en donde se presenta a las personas autoritarias con un perfil que parece incoherente: sumisas pero a la vez temerosas de parecerse a las demás. Altemeyer (1999) añade luz al déficit y a la incoherencia, señalando el recurso micro con que se procura solucionar las dificultades con que cuenta el autoritarismo para cumplir con la función de saciar la necesidad de autonomía. Analizando las respuestas a cuestionarios sobre autoritarismo y conocimiento de sí mismo, encuentra que las puntuaciones en ambas variables mantienen una relación negativa o inversa: los indivi- duos con altas puntuaciones en autoritarismo tienen un autoconocimiento muy deficitario, sustituyendo la realidad sobre su yo con imágenes idealizadas. De este modo, no hay conciencia de incoherencia y, a la vez, la idealización permite incluir en el autoconcepto la característica de individuo autónomo.

\section{Aversión IDEOLÓgICA}

Tal y como ha sido definida en el epígrafe anterior, la ideología es un acompañante inevitable. El mundo es observado siguiendo algún marco perceptivo que organiza valores y facilita la comprensión. Con los términos de McCombs (1996), las personas necesitamos contar con algún sistema de orientación que nos permita pensar y actuar en el mundo, es decir que alimente la impresión de que sabemos cómo funciona y qué hacer. Es más, generamos tal fidelidad y dependencia de estos sistemas organizados que para salvaguardarlos llegamos a admitir engaños y manipulaciones (Luhman 2005). Diversos estudios realizan observaciones en este sentido, destacando las funciones micro que se activan para mantener las adscripciones a los marcos ideológicos. Así, en una revisión de trabajos experimentales, Hafer y Bègue (2005) encuentran que las personas asumen consecuencias negativas antes que abandonar su adscripción. Las investigaciones mediante cuestionario que elabora Altemeyer (2004) señalan la coexistencia de notables incoherencias absurdas en personas con un intelecto desarrollado, elaboradas y mantenidas para mantener su ideología. Del mismo modo, Barreiro (2008) encuentra polifasia cognitiva, en su faceta de paso de pensamiento racional a mágico, a la hora de justificar algunas fidelidades ideológicas.

El carácter inevitable de las ideologías se afianza también desde su relación con las identidades individuales, de tal forma que la adscripción al marco forma parte de la construcción del Yo. No en vano, la ideología puede ser concebida como una narración sobre el mundo, mientras que la identidad es una narración sobre la propia persona, que cuenta a las demás y a sí misma (Saavedra 2007). En casos extremos, los individuos funden su identidad individual con la grupal, quedando plenamente identificados con su colectivo de referencia, tengan o no contacto físico con él (Swann y Buhrmester 2015). Es más, de modo similar a como he planteado la definición de una ideología, la identidad se concibe como tarea a la vez personal y social, mediante la que el individuo responde a tres interrogantes: quién es, quién desearía ser y quién debería ser (Rodrigo y Medina 2006). No es sorprendente, entonces, que tanto desde los estudios específicos sobre ideologías como los que versan sobre la construcción de identidades, termine existiendo algún punto de encuentro que enlaza ambas líneas de investigación (Reicher 2004; Sidanius, Pratto y Mitchell 2004; Rodríguez y Castañeda 2006). Así pues, la identidad individual 
es un constructo inevitable que permite al individuo reconocerse a sí mismo, mientras que la ideología permite a los individuos reconocer el mundo en el que transitan, a la vez que actuar en él de forma consecuente con esa visión.

No obstante y acudiendo a la mencionada categorización de Ariño (1997), concebir las ideologías como conjunto de creencias falsas o de estrategias de dominación, lleva con facilidad a sentir aversión por ellas o plantear su carácter opcional. La lógica asociada podría ser: (1) las ideologías son opcionales y dañinas; (2) se basan en valores o preferencias; luego (3) hay que prescindir de valores o preferencias y ceñir las decisiones a lo que sea "objetivamente" correcto o mejor. Tal discurso implica con facilidad un único modo de tomar buenas decisiones, justificado desde el conocimiento técnico (Orjuela 2007). Esta lógica lleva a confundir los campos de las decisiones ideológicas y las técnicas, como si cualquier aspecto de la realidad pudiera ser abordado desde cualquiera de ambos.

En la canción Los ejes de mi carreta ${ }^{1}$, Atahualpa Yupanqui responde a quienes le piden que engrase tales ejes, porque chirrían, expresando "si a mí me gusta que suenen, pa qué los quiero engrasaos". Identificar el origen del ruido y conocer la solución para que el ruido cese son dos elementos de un cuerpo de conocimientos puramente técnico. Si la decisión "es" terminar con el ruido, la actuación técnica queda clara. No obstante, esa decisión es "no-técnica", está sujeta a juicios de valor o a preferencias, como se indica en "si a mí me gusta que suenen". Multitud de decisiones cotidianas son no-técnicas, se encuentran sujetas a valor.

La aversión a las ideologías ha permitido fundamentar, siguiendo a Fair (2012), una "despolitización de la política", ofreciendo una versión supuestamente inocente, neutral y tecnocrática de las prácticas. En palabras de García-Santesmases (2002:233): "Si uno piensa que las ideologías son cosmovisiones cerradas, estrechas, que propician una adhesión fanática de sus partidarios, y provocan una tensión irreconciliable entre los defensores de las distintas posiciones, preferirá evitar cualquier programa político «excesivamente ideológico», y apostará por una «resolución técnica» de los problemas. En definitiva, preferirá delegar en los «expertos» la toma de decisiones, reservando las cuestiones de sentido para el ámbito de lo privado".

La defensa a ultranza de la objetividad o de la pureza técnica de las decisiones políticas, (como también académicas y científicas), no solo se ha denunciado como una pretensión ingenua (Barnhizer 1993), sino que también confunde frecuentemente conceptos como neutralidad, imparcialidad, objetividad, asepsia y distancia (Manzano-Arrondo 2014). En la práctica, esta defensa se concreta en discursos que presentan la decisión o actuación como inevi- table o necesaria -el agente no tiene otra opción-; que se inserta en lo que es normal o natural (Harding 1992); o bien que transita por una dimensión exclusivamente instrumental, por lo que la decisión o actuación solo puede ser juzgada en base a criterios de ejecución técnica.

La dinámica lleva desde la aversión hasta la "expertocracia", mediante tres fases cognitivas. En la primera, se acepta la especialización política, es decir, la dejación de responsabilidad de las decisiones políticas en manos de profesionales cualificados (Manzano-Arrondo 2012). En la segunda, se vacía de contenido ético a la profesión (Ball 2003), construyendo la figura de experto como alguien que actúa siguiendo criterios objetivos no sujetos a valoración ética. Y en la tercera, se ofrece la "expertocracia" como un sistema a-ideológico que permitirá tomar las mejores decisiones, objetivas y neutras (Amigot y Martínez 2015). El resultado es una opción ideológica que, si bien no tiene per se la envergadura de otras acotaciones, contiene elementos propios de los marcos ideológicos: una forma de ver el mundo, donde las ideologías son criaturas perjudiciales y evitables; una forma de preferir el mundo, en el que se opta por responsables políticos que ciñan sus actuaciones a decisiones puramente técnicas; y una forma de estar o actuar en el mundo, que implica rechazar las ideologías y apoyar a quienes señalan que toman o tomarán decisiones según patrones técnicos. Por ello, denomino a este constructo "aversión ideológica" (AVI); cuya definición sintética es 'encuadre ideológico que combina el rechazo de las ideologías y la defensa de las actuaciones políticas ceñidas a la dimensión de lo técnico'.

\section{Cuestionario para AVI}

La motivación para elaborar un instrumento de medida que dé cuenta del constructo AVI es doble. Por un lado, poner a prueba la consistencia del concepto mediante la recogida de datos. Por otro, ofrecer un cuestionario de pequeñas dimensiones que, por tanto, facilite su aplicación práctica, inclusive como una porción de un cuestionario de mayores pretensiones, inserto en investigaciones por encuesta (Burchell y Marsh 1992). Para su elaboración, procede conocer cómo se han construido los cuestionarios de los principales constructos ideológicos.

\section{Construcción de cuestionarios para la medida de marcos ideológicos}

Existe una especial abundancia de publicaciones en torno a tres marcos ideológicos concretos: el autoritarismo de ala derecha, la orientación a la dominancia social y la creencia en un mundo justo, todos ellos descritos brevemente en párrafos previos. Para estudiar el modo en que estos constructos han tomado la forma de un cuestionario, he 
acudido a la publicación original del instrumento más utilizado en cada caso.

\section{Autoritarismo de ala derecha}

Zakrison (2005) realizó abundantes modificaciones -acortamiento de expresiones, sustitución de palabras, e inversión de ítems- sobre el, hasta el momento, cuestionario más utilizado para la medida de este constructo. Aplicó el resultado, con formato de respuesta tipo Likert de acuerdo en siete puntos, a una muestra de 226 estudiantes universitarios, y consideró los 15 que mostraban una menor correlación con la orientación a la dominancia social, para incrementar la independencia de su medida de autoritarismo. Invirtió algunos ítems para conseguir siete en sentido desfavorable, y probó el resultado en una segunda muestra de 63 estudiantes universitarios, aplicando el coeficiente de consistencia interna, alfa de Cronbach, para el estudio de la fiabilidad; y la correlación lineal simple de Pearson para la relación del cuestionario con la dominancia social, obteniendo respectivamente los valores ,80 y ,26. Con una tercera muestra, de 178 estudiantes de instituto, aplicó un análisis factorial confirmatorio (AFC) para probar la estructura teórica en tres factores: agresión autoritaria, convencionalismo y sumisión autoritaria.

\section{Orientación a la dominancia social}

En su estudio de referencia, Pratto et al. (1994) utilizaron dos fuentes como bancos de ítems: el National Election Study y el S6 Conservatism Scale. Seleccionaron 70 ítems. Tras una revisión, realizaron una segunda selección hasta conseguir 14 unidades en una escala Likert de 7 puntos de acuerdo, que fueron contrabalanceadas, invirtiendo el sentido de 7 de ellas. No constan los criterios de estas dos selecciones. El cuestionario final muestra primero los 7 ítems favorables y después los desfavorables. El resultado fue probado en un total de 1952 participantes, en su gran mayoría estudiantes universitarios, provenientes de 13 muestras de diferentes orígenes y a lo largo de cinco años. Mediante un análisis factorial exploratorio (AFE) de componentes principales, comprobaron la unidimensionalidad de la escala -no consta la varianza acumulada-. Este resultado fue sometido a un AFC de máxima-verosimilitud, donde se confirma la unidimensionalidad, justificando el resultado en correlaciones estadísticamente significativas de todos los ítems con el factor -resultado muy probable con ese tamaño muestral-, y un $x^{2} / d f$ ratio de valor 2,89 . La consistencia interna media de las muestras fue de alfa $=$,83. Para el estudio de su validez, se realizaron análisis de correlación con diferentes escalas aplicadas en algunas de las muestras.

\section{Creencia en un mundo justo}

Para generar su propuesta de medida, Lipkus (1991) acudió a 402 estudiantes universitarios -52 \% mujeres, no consta edad-. Generó ítems -no consta el procedimiento- y añadió unidades de un cuestionario previo entonces muy utilizado, acudiendo a un formato Likert de acuerdo de 6 puntos. Finalmente, antes de su puesta a prueba, seleccionó siete unidades, sin especificar el procedimiento. Como consistencia interna utilizó los 21 valores de correlación entre pares, obteniendo un rango de $r=, 230$ a $r=, 577$, y un valor medio de $r=, 407$, con un alfa $=, 83$ que no mejora eliminando ningún ítem. A su vez, mediante un AFE de máxima verosimilitud, mostró un único factor con autovalor de 4,83 y $69 \%$ de varianza asociada.

\section{Criterios adoptados para este trabajo}

Tanto las herramientas originales mencionadas como sus traducciones al español -respectivamente a manos de Petit y Costa (2011), Cárdenas et al. (2010) y Barreiro, Etchezahar y Prado-Gascó (2014) - acuden a muestras de estudiantes universitarios y escalas de acuerdo tipo Likert que, salvo el trabajo más antiguo -el original de Lipkus-, utilizan el intervalo 1-7. El recurso habitual para la fiabilidad es el alfa de Cronbach y se acude a posteriores análisis factoriales, sean de corte exploratorio (AFE) o confirmatorio (AFC). A su vez, existen abundantes lagunas de información para comprender buena parte de los procesos seguidos.

Con el objetivo de aplicar un método similar a los utilizados en estos estudios mencionados, pero resolviendo algunas debilidades metodológicas, este trabajo complementa los hábitos previos con algunas sugerencias. Así, adaptando las pautas argumentadas por Morales, Urosa y Blanco (2003), se ha procurado (1) generar los ítems mediante estrategias fundamentadas, (2) realizar estudios de consistencia interna, y (3) afinar en la validez de constructo mediante AFE. Dado que AVI parte de un contenido y estructura claros, el proceso de análisis culmina poniendo a prueba el conjunto mediante AFC.

Respecto a las muestras se han considerado tres momentos: (1) una muestra de fuentes diversas para la generación de los ítems, (2) una muestra de $n=650$ participantes en un estudio on line con protagonismo de estudiantes universitarios, para la construcción del cuestionario, y (3) una muestra final de $n=340$ participantes de población general, entrevistados cara a cara, para la puesta a prueba del instrumento. Los siguientes tres epígrafes se ocupan respectivamente de ello.

\section{Generación y depuración racional de un banco inicial de ítems}

Prat y Doval (2003) aconsejan combinar la generación de ítems con criterios de experto, junto con la selección de afirmaciones de publicaciones o de narrativas. Ampliando esta sugerencia, se siguieron varios procedimientos: (1) el autor redactó un listado 
de unidades, según su propio juicio, considerando el marco teórico descrito; (2) a partir de la literatura específica sobre ideologías, se rescataron afirmaciones literales; (3) mediante entrevistas focales [50\% hombres y $50 \%$ mujeres, $50 \%$ con estudios universitarios, edades comprendidas entre los 21 y los 77 años, con un total de 18 horas de audio] que versaban sobre la forma de construir una imagen sobre el funcionamiento del mundo, se identificaron afirmaciones literales y se redactaron algunas afirmaciones que sintetizaban respuestas demasiado extensas como para ser susceptibles de formar ítems de manera literal; y (4) aprovechando un estudio de panel, on line, se solicitó a los participantes [ $\mathrm{n}=58$, en la fase en que se utilizó este recurso] que emitieran afirmaciones que contuvieran la palabra "ideología" o que hicieran referencia indirecta al concepto. Los procedimientos 1 y 2 garantizan redacciones pertinentes según los marcos teóricos. Los procedimientos 3 y 4 buscan expresiones "naturales", es decir, generadas desde muestras similares a las que pueden utilizarse posteriormente para validar el cuestionario.

El resultado fue motivo de una primera depuración, eliminando redacciones coincidentes o equivalentes, lo que derivó en un listado inicial de 62 ítems potenciales. Este listado se organizó en tres grupos: consideración negativa de las ideologías, defensa de actuaciones políticas a-ideológicas, y concepción inevitable o respetuosa de las ideologías (que se contrapone a los dos grupos anteriores). Finalmente, el listado se envió a seis expertos, seleccionados por haber realizado al menos una publicación en torno a cualesquiera de los siguientes constructos: ideología, actitud, identidad o necesidades. Junto con el listado, se les remitió un borrador de este artículo, conteniendo el marco teórico. Su trabajo consistió en agrupar los ítems en tres compartimentos: claramente relacionados con el concepto específico, claramente no relacionados y de clasificación dudosa. Se consideraron únicamente los ítems con 5 o 6 coincidencias en el compartimento "claramente relacionados", y aquellos con 3 o 4 coincidencias en ese compartimento, que ninguno de los restantes expertos había situado en "claramente no relacionados". Solo 35 unidades cumplieron estos criterios. El Apéndice 1 lista los ítems no considerados, mientras que la Tabla 1 hace otro tanto con los enunciados seleccionados.

\section{Estudio 1. Depuración de ítems y propuesta de cuestionario}

Considerando la discusión de Morales (2000) sobre el recurso de un número par o impar de opciones y el formato habitual utilizado en las investigaciones sobre marcos ideológicos mencionadas en los epígrafes previos, se optó finalmente por utilizar 7 grados de acuerdo. Para facilitar la situación de respuesta, el cuestionario contiene el siguiente en- cabezado: "Imagine que pasea usted por una sala donde se encuentra mucha gente. Conversan sobre política e ideologías. Lo que sigue a continuación es un listado de afirmaciones realizadas en la sala, y dispuestas por orden alfabético".

La selección de ítems mantuvo en todo momento no solo el objetivo de conseguir un buen instrumento para AVI, sino también para cada una de sus dos escalas: rechazo de las ideologías (RID) y preferencia por decisiones políticas técnicas (PDT). Por ello, los análisis se realizaron tanto con el listado total de ítems, como dentro de cada uno de los dos conjuntos específicos. Por otro lado, se buscó una reducción drástica del número de unidades, para ofrecer una herramienta fácil de manejar. Este proceso derivó finalmente en un instrumento de diez ítems, cinco por escala. Para llegar a ello, se operó en tres momentos: (1) estudio de la consistencia interna para una selección inicial de unidades con altos valores de correlación ítem-total corregida; (2) AFE, muy especialmente para considerar las comunalidades iniciales y un resultado coherente en la matriz de configuración; y (3) AFC para seleccionar finalmente las unidades con mejores cargas factoriales y que procuran un buen ajuste del modelo.

\section{Participantes}

El cuestionario se dispuso on line, mediante el recurso de Google Formularios, incluyendo al inicio del mismo una breve indagación sobre edad, sexo y nivel de estudios. Gracias a personal colaborador - estudiantes de prácticas de investigación en primer y último curso del grado de psicología- se aplicaron dos procedimientos para la captación de participantes. Por un lado, el personal colaborador compartió el enlace del cuestionario en sus respectivas redes de Facebook y Twitter. Por otro, realizaron invitaciones expresas a personas mayores de 30 años, para estimular la participación de estudiantes no universitarios. Ambos procedimientos dieron lugar a una muestra de $n=703$, completada durante los meses de septiembre y octubre de 2016. Se eliminaron los registros con más de un $10 \%$ de datos ausentes, dos casos con edades inferiores a 18 años, y todos los que no habían respondido a la edad. De la muestra resultante, $n=650$, la edad media fue de 31,92 años (desviación tipo: 12,43); $64 \%$ mujeres; $56 \%$ con estudios superiores, $35 \%$ secundarios y $9 \%$ primarios.

El tamaño muestral cumple sobradamente los criterios revisados por Frías-Navarro y Pascual en 2012 para la realización de un AFE: comúnmente no menos de 100 participantes en total, ni menos de 10 por ítem.

\section{Consistencia interna}

Se realizó mediante el recurso habitual del alfa de Cronbach, prescindiendo de las unidades con una correlación ítem-total corregida inferior a $r_{\text {cor }}=, 33$ (lo 
Tabla 1.

Listado de ítems para la versión preliminar del cuestionario AVI.

\begin{tabular}{|c|c|}
\hline \multicolumn{2}{|r|}{ RID: rechazo de las ideologías } \\
\hline 1 & Cuando me informo, escojo medios sin ideología. \\
\hline 4 & Es bueno tener una ideología (I). \\
\hline 5 & Es fundamental tener una ideología en la vida (I). \\
\hline 6 & Es imposible vivir sin ideología (I). \\
\hline 7 & Es mejor mantenerse al margen de las ideologías. \\
\hline 14 & Las ideologías ayudan a interpretar las cosas que ocurren (I). \\
\hline 15 & Las ideologías engañan sobre cómo funciona el mundo. \\
\hline 16 & Las ideologías no sirven para nada. \\
\hline 17 & Las ideologías son las grandes culpables de los males del mundo. \\
\hline 18 & Las ideologías son para los radicales. \\
\hline 21 & Me gusta relacionarme con personas sin ideología. \\
\hline 24 & No me gustan las ideologías. \\
\hline 25 & No tengo ideología. \\
\hline 27 & Para vivir en una sociedad mejor habría que suprimir las ideologías. \\
\hline 29 & Quien siga una ideología es que no tiene personalidad. \\
\hline 31 & Tener una ideología es un error. \\
\hline 32 & Todas las ideologías se equivocan. \\
\hline 33 & Todo el mundo tiene alguna ideología. \\
\hline \multicolumn{2}{|r|}{ PDT: preferencia por decisiones políticas exclusivamente técnicas } \\
\hline 2 & Cuando voto a un candidato, espero que gobierne sin ideología. \\
\hline 3 & El mundo es como es y no según cómo se mire. \\
\hline 8 & Es un error que los políticos tomen decisiones ideológicas. \\
\hline 9 & Escojo votar a partidos que no sigan ninguna ideología. \\
\hline 10 & Gobernar debería consistir en tomar decisiones exclusivamente técnicas. \\
\hline 11 & Hay que andar por la vida viendo las cosas tal y como son. \\
\hline 12 & La política debe ser independiente de la ideología. \\
\hline 13 & Las discusiones parlamentarias deberían ceñirse a lo técnico. \\
\hline 19 & Las leyes han de ser independientes de la ideología de quien las decide. \\
\hline 20 & Los políticos deberían tomar solo decisiones técnicas. \\
\hline 22 & Me sentiría mejor si gobernaran los técnicos en lugar de los políticos. \\
\hline 23 & Me siento mejor pensando que las leyes se redactan con criterios exclusivamente técnicos. \\
\hline 26 & Para hacer bien su trabajo, un político necesita tener una ideología (I). \\
\hline 28 & Prefiero el político práctico al que sigue una ideología. \\
\hline 30 & Solo hay una forma correcta de ver las cosas. \\
\hline 34 & Tomar decisiones según una ideología es dejarse arrastrar por los sentimientos. \\
\hline 35 & Un cargo político no debería tener ideología. \\
\hline
\end{tabular}

que implica una variación lineal compartida límite del $10 \%)$, en la escala específica o en la general. Previamente, se invirtió el valor de los ítems no favorables: 4, 5, 6, 14, 26 у 33 .

Como resultado, el cuestionario quedó reducido a 28 unidades, 14 por escala. Se prescindió de los ítems 1 ( $r_{\text {cor }}=, 316$ en RID), $3\left(r_{\text {cor }}=, 222\right.$ en AVI y ,241 en PDT), 6 ( $r_{\text {cor }}=, 281$ en AVI y ,324 en RID), $11\left(r_{\text {cor }}=, 324\right.$ en AVI), 30 ( $r_{\text {cor }}=, 225$ en AVI y ,207 en PDT), 32 ( $r_{\text {cor }}=312$ en AVI y ,327 en RID) y 33 $\left(r_{\text {cor }}=, 130\right.$ en AVI y, 175 en RID). Los valores finales para el alfa de Cronbach fueron RID = ,886; PDT = ,906; y $\mathrm{AVI}=$, ,941.

\section{Análisis factorial exploratorio}

Se recurrió al método de ejes principales como procedimiento de extracción. Debido a que considera únicamente la variabilidad común de los ítems, frente a la totalidad de la extracción por componentes principales (Pérez y Medrano 2010), no solo es coherente con la motivación de extraer factores comunes, sino que hace más exigente la selección de 
unidades cuando se acude al criterio de no admitir aquellas con una comunalidad inicial inferior a ,5. En línea con la relación teórica que se espera entre las dos escalas del constructo se optó por el método de rotación oblimin, con el referente delta $=0$.

Para la escala RID, las comunalidades insuficientes se corresponden con los ítems $7(, 324), 14$ (,161), $15(, 267), 17(, 342), 21(, 332)$ y $29(, 371)$. Al realizar un nuevo AFE con los ocho ítems restantes, se obtiene un factor principal con autovalor 4,198 y varianza acumulada del 52,48 \%. El siguiente factor cuenta con un autovalor limítrofe $(1,194)$, que ya en la matriz factorial sin acumular muestra claramente su relación con dos únicos ítems, los dos invertidos o desfavorables que restan en el conjunto: 4 y 5 . Al eliminarlos del análisis, el conjunto de ítems para esta escala queda con las 6 unidades que se muestran en la Tabla 2.

Para la escala PDT, las comunalidades insuficientes se corresponden con los ítems $2(, 385), 8(, 384)$, $19(, 403), 26(, 233)$ y 34 (,269). En un siguiente AFE, la comunalidad del ítem 9 cae a ,383. Los ocho ítems restantes, junto con los seis de RID, son utilizados para un AFE de escala conjunta. Los resultados muestran con claridad dos factores (con un 61,86 \% de varianza total considerada) que se corresponden con los ítems de ambas escalas, a excepción de las unidades 12 y 35 , cuyos valores en la matriz de configuración no cumplen con el criterio de estructura simple (Brown 2009): en valores absolutos, sus aportes respectivos son de ,312 y ,509 para el primer factor $\mathrm{y}, 454$ y ,316 para el segundo. Al excluirlos del análisis, el resultado muestra una estructura simple, cuyos detalles se incluyen en la Tabla 2 (columnas $\mathrm{Cl}$ para las comunalidades, y fRID y fPDT para los valores de la matriz de configuración que resulta de la rotación).

Finalmente, se aplicó un AFC con el método de estimación DWLS (Diagonally Weithted Least Square). Los resultados de las simulaciones realizadas por Baghdarnia, Firozy y Gorji (2014) y Li (2016) muestran que este procedimiento es más aconsejable que el habitual de máxima verosimilitud a la hora de operar con variables ordinales, como las que provienen de las escalas tipo Likert. Si bien el resultado final es aceptable, el ítem 31 no muestra una carga factorial totalmente satisfactoria $(, 63<, 7)$. Este comportamiento limítrofe no es grave. Sin embargo, se mantiene en el resto de características: un valor medio muy bajo $(2,03)$ con la menor variabilidad del conjunto $(1,37)$, correlaciones ítem-total satisfactorias pero también las más bajas del conjunto, y una comunalidad respecto al AFE del total de la escala también relativamente baja $(, 428<, 5)$. Con el objetivo de seguir reduciendo la escala, procedía no solo eliminar este ítem, sino también otro de PDT, manteniendo el equilibrio entre el peso de ambas en el cuestionario final. La Tabla 2 señala al ítem 28 como el más idóneo: de nuevo variabilidad, correlación ítem-total, comunalidad AFE y carga factorial AFC con valores más bajos que el resto de los ítems de su escala específica. La Tabla 3 muestra el nuevo resultado, que conforma la propuesta final para el instrumento de medida de AVI.

Tabla 2.

Resultados para el cuestionario AVI de 12 unidades, con muestra on line.

\begin{tabular}{|c|c|c|c|c|c|c|c|c|c|c|}
\hline & & $M$ & SD & ESP & TOT & $\mathrm{Cl}$ & fRID & fPDT & cRID & cPDT \\
\hline \multirow{6}{*}{$\frac{\rho}{\bar{x}}$} & 16 & 2,50 & 1,55 &, 71 & ,62 & ,459 & ,68 & ,06 & ,71 & ,00 \\
\hline & 18 & 2,37 & 1,61 &, 75 &, 59 & ,466 & ,71 & ,00 & ,69 & ,00 \\
\hline & 24 & 3,08 & 1,66 &, 76 & ,67 & ,595 & ,80 & ,02 & ,77 & ,00 \\
\hline & 25 & 2,63 & 1,73 & ,69 &, 59 & ,503 & ,74 &,- 02 & ,69 & ,00 \\
\hline & 27 & 2,90 & 1,65 &, 75 & ,66 & ,503 & ,63 & , 16 & ,78 & ,00 \\
\hline & 31 & 2,03 & 1,37 & ,62 & ,54 & ,428 & ,72 &,- 07 & 63 & ,00 \\
\hline \multicolumn{2}{|r|}{ Total } & 2,57 & 1,23 & ,89 & & & & & & \\
\hline \multirow{6}{*}{$\stackrel{\llcorner}{\circ}$} & 10 & 3,40 & 1,86 & ,66 & ,62 & ,562 &,- 10 & ,82 & ,00 & ,71 \\
\hline & 13 & 3,84 & 1,85 & ,66 & 67 & ,607 &,- 06 & ,85 & ,00 & ,76 \\
\hline & 20 & 3,74 & 1,82 &, 74 & ,73 & 641 & ,07 & ,78 & ,00 & ,83 \\
\hline & 22 & 4,12 & 1,90 & ,66 & 68 & ,548 &, 13 & ,66 & ,00 & ,76 \\
\hline & 23 & 4,05 & 1,77 & ,66 & 68 & ,587 &,- 01 & ,80 & ,00 & ,77 \\
\hline & 28 & 4,44 & 1,73 & ,63 & 63 & ,451 & ,19 &, 54 & ,00 & ,70 \\
\hline \multicolumn{2}{|r|}{ Total } & 3,93 & 1,47 &, 87 & & & & & & \\
\hline \multicolumn{2}{|c|}{ G.Total } & 3,24 & 1,21 & & ,91 & & & & & \\
\hline
\end{tabular}

M: media; SD: desviación tipo; ESP: correlación ítem-total corregida para la escala específica; TOT: idem para la escala total; CI: comunalidades iniciales para la escala total; fRID y fPDT: valores de la matriz de configuración tras la rotación oblimin; cRID y cPDT: cargas factoriales en el AFC. 
Tabla 3.

Resultados para el cuestionario AVI de 12 unidades, con muestra on line.

\begin{tabular}{|c|c|c|c|c|c|c|c|c|c|c|}
\hline & & $M$ & SD & ESP & TOT & $\mathrm{Cl}$ & fRID & fPDT & CRID & cPDT \\
\hline \multirow{5}{*}{$\stackrel{\underline{\bar{x}}}{\underline{\underline{\alpha}}}$} & 16 & 2,50 & 1,55 & ,66 & ,61 & ,451 & ,03 & ,70 & ,71 & ,00 \\
\hline & 18 & 2,37 & 1,61 & ,64 & ,58 & ,431 & ,02 & 68 & ,68 & ,00 \\
\hline & 24 & 3,08 & 1,66 & ,74 & ,66 & ,586 &,- 05 & ,86 & ,77 & ,00 \\
\hline & 25 & 2,63 & 1,73 & ,66 &, 58 & ,493 &,- 05 & ,76 & ,69 & ,00 \\
\hline & 27 & 2,90 & 1,65 & ,63 & ,64 & ,450 & ,16 & ,60 &, 76 & ,00 \\
\hline \multicolumn{2}{|r|}{ Total } & 2,69 & 1,29 & 85 & & & & & & \\
\hline \multirow{5}{*}{$\stackrel{\leftarrow}{\circ}$} & 10 & 3,40 & 1,86 & ,72 & ,63 & ,556 & ,84 &,- 10 & ,00 & ,73 \\
\hline & 13 & 3,84 & 1,85 & ,74 & ,67 & ,598 & 84 &,- 05 & ,00 & ,77 \\
\hline & 20 & 3,74 & 1,82 & ,77 &, 74 & 637 &, 77 & , 10 & ,00 & 84 \\
\hline & 22 & 4,12 & 1,90 & ,67 & ,67 &, 531 & ,60 & , 18 & ,00 &, 75 \\
\hline & 23 & 4,05 & 1,77 & ,74 & ,68 & ,574 &, 75 & ,04 & ,00 & ,77 \\
\hline \multicolumn{2}{|r|}{ Total } & 3,82 & 1,53 & ,89 & & & & & & \\
\hline \multicolumn{2}{|c|}{ G.Total } & 3,24 & 1,25 & & 90 & & & & & \\
\hline
\end{tabular}

M: media; SD: desviación tipo; ESP: correlación ítem-total corregida para la escala específica; TOT: idem para la escala total; Cl: comunalidades iniciales para la escala total; fRID y fPDT: valores de la matriz de configuración tras la rotación oblimin; cRID y cPDT: cargas factoriales en el AFC.

En definitiva, se ofrece un cuestionario de 10 ítems, 5 por escala, con una consistencia interna de valores RID = ,85; PDT = ,89; y AVI = ,90; tras obtener dos factores de un AFE con autovalores 6,172 y 1,444 (el siguiente fue de ,658), que responden del $63,46 \%$ de la varianza, y mantienen una correlación entre ambos con valor $r=, 61 ; \mathrm{KMO}=, 927$ y prueba de esfericidad de Barlett de $X^{2}=4079, \mathrm{gl}=66$ y $p<, 001$. EI AFC muestra cargas no inferiores a ,7 (redondeo de un dígito decimal) y parámetros con valores de buen ajuste, siguiendo los estándares $\operatorname{RMSEA}(, 030)$ y $\operatorname{SRMR}(, 042)$ inferiores a ,08; a la vez que TLI $(, 995)$ y CFI $(, 996)$ superiores a ,95.

\section{Estudio 2. Comprobación del cuestionario en una muestra de población general}

Con el objetivo de poner el instrumento a prueba en una muestra no proveniente de estudiantes universitarios, se aplicó a población general, comprobando de nuevo su estructura emergente desde un AFE y confirmando el modelo a través de un AFC con las mismas características procedimentales indicadas en el epígrafe anterior.

Se obtuvo una muestra de tamaño $n=340$, mediante cuotas de edad y sexo, y encuesta cara a cara en la provincia de Sevilla, a población mayor de edad. Para el cálculo de las cuotas se utilizó el padrón continuo a 1 de enero de 2013, último disponible en la base pública del Instituto Nacional de Estadística de España. La recogida de datos se llevó a cabo entre finales de octubre e inicios de noviembre de 2016. El cuestionario, además del texto introductorio y algunas preguntas de carácter sociodemográfico, contenía los ítems seleccionados, dispuestos por orden alfabético, según se muestra en la Tabla 4. Los resultados del análisis son sintetizados en la Tabla 5.

Los resultados para la muestra de población general en muestreo cara a cara, confirman los obtenidos en el estudio previo, con un comportamiento muy similar. Se observa una buena consistencia interna en cada escala específica y en el conjunto, así como valores suficientemente elevados para las comunalidades, la matriz de configuración AFE y las cargas factoriales AFC. Los autovalores en el AFE fueron de 4,658 y 2,391 (,597 para el siguiente), que responden del $70,5 \%$ de la varianza, y mantienen una correlación entre ambos con valor $r=, 35 ; \mathrm{KMO}=, 873$ y prueba de esfericidad de Barlett de $X^{2}=1945, \mathrm{gl}=45$ y $p<, 001$. Los parámetros de ajuste para el AFC fueron RMSEA $=, 040 ;$ SRMR $=, 055 ; \mathrm{TLI}=, 991 ; \mathrm{y} \mathrm{CFI}=, 993$.

\section{Utilidad de la variabilidad}

Un aspecto interesante en cualquier instrumento de medida es su capacidad de discriminación. En los objetivos de este trabajo, por razones de extensión, no se ha incluido realizar un estudio expreso para validar el constructo mediante su relación con otros conceptos potencialmente relacionados. En tal caso, procedería realizar análisis no solo de relación - directa o inversa-, sino también de discriminación, para observar que las puntuaciones en AVI permiten discriminar entre valores distintos en tales conceptos relacionados. No obstante, dado que el potencial de discriminación se encuentra en la variabilidad de las puntuaciones -una medida que no varía, no correlaciona con ninguna otra-, una medida indirecta 
Tabla 4.

Relación de ítems para el cuestionario AVI.

\begin{tabular}{|c|c|c|c|}
\hline \# & Enunciado & RID & PDT \\
\hline 1 & Gobernar debería consistir en tomar decisiones exclusivamente técnicas. & & $\mathrm{x}$ \\
\hline 2 & Las discusiones parlamentarias deberían ceñirse a lo técnico. & & $\mathrm{X}$ \\
\hline 3 & Las ideologías no sirven para nada. & $\mathrm{x}$ & \\
\hline 4 & Las ideologías son para los radicales. & $\mathrm{x}$ & \\
\hline 5 & Los políticos deberían tomar solo decisiones técnicas. & & $\mathrm{x}$ \\
\hline 6 & Me sentiría mejor si gobernaran los técnicos en lugar de los políticos. & & $\mathrm{X}$ \\
\hline 7 & Me siento mejor pensando que las leyes se redactan con criterios exclusivamente técnicos. & & $\mathrm{x}$ \\
\hline 8 & No me gustan las ideologías. & $\mathrm{x}$ & \\
\hline 9 & No tengo ideología. & $x$ & \\
\hline 10 & Para vivir en una sociedad mejor habría que suprimir las ideologías. & $\mathrm{x}$ & \\
\hline
\end{tabular}

Tabla 5.

Resultados para el cuestionario AVI de 10 unidades, con población general.

\begin{tabular}{|c|c|c|c|c|c|c|c|c|c|c|}
\hline & & M & SD & ESP & TOT & $\mathrm{Cl}$ & fRID & fPDT & cRID & CPDT \\
\hline \multirow{5}{*}{ 음 } & 16 & 2,83 & 1,92 & ,74 & ,66 & ,601 & ,09 & ,76 & 84 & 00 \\
\hline & 18 & 2,58 & 1,86 & ,68 & 58 & ,483 & ,06 & ,69 & ,74 & 00 \\
\hline & 24 & 3,08 & 1,93 & ,83 & 60 & ,726 &,- 13 & ,95 & ,81 & ,00 \\
\hline & 25 & 2,82 & 2,08 & ,75 & ,58 & ,593 &,- 04 & ,81 & ,78 & ,00 \\
\hline & 27 & 2,96 & 1,94 & ,74 & ,63 & ,575 & ,05 & ,77 & ,79 & ,00 \\
\hline & Total & 2,86 & 1,65 & ,90 & & & & & & \\
\hline \multirow{5}{*}{ 占 } & 10 & 3,50 & 1,77 & ,75 & 58 & ,615 & ,82 & ,00 & ,00 & ,81 \\
\hline & 13 & 3,64 & 1,79 & ,72 & 53 & ,591 & ,81 &,- 06 & ,00 & ,75 \\
\hline & 20 & 3,41 & 1,78 & ,77 & 56 & ,635 & ,88 &,- 07 & ,00 & ,80 \\
\hline & 22 & 3,94 & 1,93 & ,65 & ,61 & ,497 & ,63 & ,17 & ,00 & ,76 \\
\hline & 23 & 3,76 & 1,78 & ,73 & 58 & ,568 & ,77 & ,03 & ,00 & ,78 \\
\hline & Total & 3,65 & 1,50 & ,89 & & & & & & \\
\hline \multicolumn{2}{|c|}{ G.Total } & 3,25 & 1,30 & & 87 & & & & & \\
\hline
\end{tabular}

M: media; SD: desviación tipo; ESP: correlación ítem-total corregida para la escala específica; TOT: idem para la escala total; Cl: comunalidades iniciales para la escala total; fRID y fPDT: valores de la matriz de configuración tras la rotación oblimin; cRID y cPDT: cargas factoriales en el AFC.

es estudiar la variabilidad interna del constructo. La Tabla 6 muestra los resultados sobre este asunto, respecto a los dos componentes y la medida general, en ambas muestras. La desviación tipo acotada -cuyo mecanismo de cálculo es justificado en Scurtu, Manzano-Arrondo y Rodríguez (2016)- expresa el valor de la desviación tipo en el intervalo $(0,100)$, indicando respectivamente la mínima y la máxima variabilidad posibles considerando el recorrido de la variable y el valor de la media aritmética. Los resultados muestran valores de variabilidad que rondan el $50 \%$ del máximo posible y, por tanto, permiten esperar capacidad de discriminación en la utilización del cuestionario $\mathrm{AVI}$, sea en general o por componentes.

\section{Discusión Y CONCLUSIONES}

La definición propuesta para el concepto de ideología focaliza el interés en dos aspectos: el carácter organizativo y holístico de un marco ideológico, y su
Tabla 6.

Promedios y variabilidad.

\begin{tabular}{|c|l|c|c|c|}
\hline & & $\mathrm{M}$ & $\mathrm{SD}$ & $\mathrm{VA}$ \\
\hline \multirow{4}{*}{ On line } & $\mathrm{RID}$ & 2,69 & 1,29 & 48 \\
\cline { 2 - 5 } & $\mathrm{PDT}$ & 3,82 & 1,53 & 51 \\
\cline { 2 - 5 } & $\mathrm{AVI}$ & 3,24 & 1,25 & 43 \\
\hline \multirow{4}{*}{ Cara a cara } & $\mathrm{RID}$ & 2,86 & 1,65 & 59 \\
\cline { 2 - 5 } & PDT & 3,65 & 1,50 & 50 \\
\cline { 2 - 5 } & AVI & 3,25 & 1,30 & 45 \\
\hline
\end{tabular}

M: media; SD: desviación tipo; VA: SD acotada.

papel fundamental para orientar el pensamiento y la acción de los individuos. El componente "una forma de ver el mundo" implica recursos para interpretarlo. Con "una forma de preferir el mundo" se incluyen los valores y los juicios de valor. Y mediante "una forma de actuar en el mundo" se conectan las visiones y juicios estructurales con las acciones individuales. El 
mundo ideológico es social, se refiere al modo en que las personas se organizan en un todo amplio, en cómo es bueno -o malo- que funcione ese todo y en qué han de hacer -o evitar-. En marcos ideológicos previamente descritos se observa con claridad ese papel de orientación y protección individual. En el autoritarismo, se propone la sumisión a -y defensa de- la autoridad. En la creencia en un mundo justo, cada cual puede controlar totalmente su futuro mediante un comportamiento presente adecuado. En la dominancia social, cada individuo ha de preocuparse de sí mismo en un mundo de desequilibrios sociales naturales. En el yoísmo (Manzano-Arrondo 2015), cada cual ha de diseñar sus propios proyectos vitales, considerando que otros individuos harán lo mismo y exigiendo para todo ello suficientes garantías normativas e institucionales. La certidumbre que implica una función definida para el Yo, inserta en un funcionamiento acotado para el mundo, colabora en la comprensión de la fidelidad ideológica.

En este marco discursivo, el artículo acota un enfoque ideológico, es decir, una forma de ver, preferir y actuar, denominado Aversión Ideológica, y definido en términos de rechazo a la mediación de las ideologías en la dimensión de las decisiones políticas. Los resultados han surgido de dos muestras independientes, suministrando un comportamiento similar, lo que permite una aceptable seguridad en las conclusiones. En ambas ocasiones se han observado niveles medios para el componente "Preferencia por decisiones políticas exclusivamente técnicas" -frente a un centro de recorrido de valor 4 , las

\section{NOTAS}

1. Milonga con música de Atahualpa Yupanqui y letra de Romildo Risso.

\section{Referencias Bibliográficas}

Adorno, Th. W., E. Frenkel-Brunswik, D. J. Levinson y R. N. Sanford. 1950. The authoritarian personality. New York: Norton and Company.

Altemeyer, B. 1999. "To thine own self be untrue: self-awareness in authoritarians". North American Journal of Psychology 1: 157-164.

Altemeyer, B. 2004. "Highly dominating, highly authoritarian personalities". The Journal of Social Psychology 144: 421-447. DOI: http://dx.doi.org/10.3200/ SOCP.144.4.421-448

Amigot, P. y L. Martínez. 2015. "Procesos de subjetivación en el contexto neoliberal. El caso de la evaluación del profesorado y la investigación universitaria“. RASE 8: 137-154.

André, J. 1985. "Power, oppression and gender". Social Theory and Practice 11: 107-122. DOI: http://dx.doi. org/10.5840/soctheorpract19851116

Apple, M. W. 2011. "Global crises, social justice, and teacher education“. Journal of Teacher Education, 62, 222-234. DOI: http://dx.doi.org/10.1177/0022487110385428

Ariño, A. 1997. "Ideologías, discursos y dominación". REIS 79: 197-219. muestras han suministrado medias de valores 3,82 y 3,65- y medio-bajos para el componente "Rechazo de las ideologías" -2,69 y 2,86-. En su conjunto, implicaría cierto nivel de tolerancia en general para la mediación de las ideologías, pero menos transigencia en el caso de su presencia en las decisiones políticas. Este resultado refuerza la especificidad del constructo: no se refiere tanto a una aversión generalizada, sino también y especialmente a un rechazo específico. La clara relación entre los dos componentes y los altos valores de consistencia interna total, forman parte también de la coherencia interna de la propuesta. No obstante, las dos muestras han suministrado valores claramente diferentes de correlación entre los dos componentes -,67 para la muestra on line; y ,35 para la recogida cara a cara-, lo que no permite establecer conclusiones generales para la cuantía de la relación interna y merecería replicaciones del estudio.

El cuestionario propuesto cuenta con buenas características psicométricas, incluyendo suficiente diversidad en las puntuaciones obtenidas de los participantes, como para suponer que será un instrumento útil en el estudio directo de $\mathrm{AVI}$ y de su relación con otros constructos. Esta utilidad ha mediado todo el proceso de construcción de la herramienta, buscando, entre otros motivos, una dimensión manejable de la herramienta. No obstante, como ocurre con todo instrumento nuevo, la utilidad real requiere mayores aportes empíricos en muestras de contextos diversos y también en relación a un conjunto amplio de conceptos potencialmente relacionados.
Baghdarnia, M., R. Firozy y R. Gorji. 2014. "The comparison of two methods of maximum likelihood (ML) and diagonally weighted least squares (DWLS) in testing construct validity of achievement goals". Journal of Educational and Management Studies 4: 22-38.

Ball, St. J. 2003. "Profesionalismo, gerencialismo y performatividad“. Revista Educación y Pedagogía 15: 87-104.

Barnhizer, D. 1993. "Freedom to do what? Institutional neutrality, academic freedom, and academic responsibility". Journal of Legal Education 43: 346-357.

Barreiro, A. 2008. "El desarrollo de la creencia en un mundo justo: relaciones entre la construcción individual del conocimiento y los saberes producidos colectivamente“. Estudios de Psicología 29: 289-299. DOI: http://dx.doi.org/10.1174/021093908786145403

Barreiro, A., E. Etchezahar y V. Prado-Gascó. 2014. "Creencia global en un mundo justo: validación de la escala de Lipkus en estudiantes universitarios de la ciudad de Buenos Aires". Interdisciplinaria 31: 57-71.

Block, J. y J. H. Block. 2006. "Nursery school personality and political orientation two decades later". Journal of Research in Personality 40: 734-749. DOI: http://dx.doi. org/10.1016/j.jrp.2005.09.005 
Bocock, R. 1995. El consumo. Madrid: Talasa Ediciones.

Brown, J. D. 2009. "Choosing the Right Type of Rotation in PCA and EFA". Shiken: JALT Testing y Evaluation SIG Newsletter 13: 20-25.

Brussino, S., H. H. Rabbia, D. Imhoff y A. P. Paz. 2011. "Dimensión operativa de la ideología política en ciudadanos de Córdoba - Argentina“. Psicología Política 43: 85-106.

Burchell, Br. y C. Marsh. 1992. "The effect of questionnaire length on survey response". Quality and Quantity 26: 233-244. DOI: http://dx.doi.org/10.1007/BF00172427

Cárdenas, M., P. Meza, K. Lagues y S. Yañez. 2010. "Adaptación y validación de la Escala de Orientación a la Dominancia Social SDO) en una muestra chilena“. Universitas Psychologica 9: 161-168.

Chatard, A. y L. Selimbegovic. 2008. "The intergenerational transmission of social dominance: a three-generation study“. European Journal of Personality 22: 541-551. DOI: http://dx.doi.org/10.1002/per.684

Deci, E. L. y R. M. Ryan. 2000. "The 'What' and 'Why' of goal pursuits: human needs and the Self-Determination Theory“. Psychological Inquiry 11: 227-268. DOI: http:// dx.doi.org/10.1207/S15327965PLI1104_01

(van) Dijk, T. A. 2003. "La multidisciplinariedad del análisis crítico del discurso: un alegato en favor de la diversidad“. Pp. 143-177 en Métodos de análisis crítico del discurso, editado por R. Wodak y M. Meyer. Barcelona: Gedisa.

Deutsch, M. 2006. "A framework for thinking about oppression and its change". Social Justice Research 19: 7-41. DOI: http://dx.doi.org/10.1007/s11211-006-9998-3

Duckitt, J. y K. Fisher. 2003. "The impact of social threat on worldview and ideological attitudes". Political Psychology 24: 199-222. DOI: http://dx.doi.org/10.1111/0162$895 \times .00322$

Eagleton, T. 1997. Ideología. Una introducción. Barcelona: Paidós.

Esquivel, J. E. 2007. "Chile: campo experimental para la reforma universitaria“. Perfiles Educativos 29: 41-59.

Fair, H. 2012. "El discurso político de la antipolítica“. Razón y Palabra 80: 1-20.

Freire, P. 2001. Pedagogía de la indignación. Madrid: Morata.

Furnham, A. 2002. "Belief in a just world: research progress over the past decade". Personality and Individual Differences 34: 795-817. DOI: http://dx.doi.org/10.1016/ S0191-8869(02)00072-7

Garcés, J. 1988. Valores humanos. Principales concepciones teóricas. Valencia: Nau Llibres.

García-Castro, J. D. 2010. "Ideología de la desigualdad: análisis de la investigación empírica en psicología social". Revista Electrónica de Psicología Política 8: 67-87.

García-Santesmases, A. 2002. "Estado, mercado y sociedad civil". Pp. 217-234 en Filosofía política: ideas políticas y movimientos sociales, editado por F. Quesada. Madrid: Trotta.

Hafer, C. L. y L. Bègue. 2005. "Experimental research on just-world theory: problems, developments, and future challenges". Psychological Bulletin 131: 128-167. DOI: http://dx.doi.org/10.1037/0033-2909.131.1.128

Harding, S. 1992. "After the neutrality ideal: science, politics, and 'strong objectivity'". Social Research 59: 567-587.

Jost, J. T. 2006. "The end of the end of ideology". American Psychologist 61: 651-670. DOI: http://dx.doi. org/10.1037/0003-066X.61.7.651

Jost, J. T., M. R. Banaji y Br. A. Nosek. 2004. "A decade of system justification theory: accumulated evidence of conscious and unconscious bolstering of the status quo".
Political Psychology 25: 881-919. DOI: http://dx.doi. org/10.1111/j.1467-9221.2004.00402.x

Jost, J. T., S. Blount, J. Pfeffer y G. Hunyady. 2003. "Fair market ideology: its cognitive-motivational underpinnings". Research in Organizational Behavior 25, 53-91. DOI: http://dx.doi.org/10.1016/S0191-3085(03)25002-4

Jost, J. T., Chr. M. Federico y J. L. Napier. 2009. "Political ideology: its structure, functions, and elective affinities". Annual Review of Psychology 60: 307-337. DOI: http:// dx.doi.org/10.1146/annurev.psych.60.110707.163600

Jost, J.T. y Thompson, E.P. 1999. "Group-Based Dominance and Opposition to Equality as Independent Predictors of Self-Esteem, Ethnocentrism, and Social Policy Attitudes among African Americans and European Americans". Journal of Experimental Social Psychology 36: 209-232. DOI: http://dx.doi.org/10.1006/jesp.1999.1403

Kay, A. C., J. A. Whitson, D. Gaucher y A. D. Galinsky. 2009. "Compensatory control. Achieving order through the mind, our institutions, and the heavens". Current Directions in Psychological Science 18: 264-268. DOI: http://dx.doi.org/10.1111/j.1467-8721.2009.01649.x

Knight, K. 2006. "Transformations of the concept of ideology in the twentieth century". American Political Science Review 100: 619-626. DOI: http://dx.doi.org/10.1017/ S0003055406062502

Kossovska, M. 2003. "The relationship between need for closure and conservative beliefs in Western and Eastern Europe“. Political Psychology 24: 501-518. DOI: http:// dx.doi.org/10.1111/0162-895X.00338

Kugler, M., J. T. Jost y Sh. Noorbaloochi. 2014. "Another look at moral foundations theory: do Autoritarianism and Social Dominance Orientation explain LiberalConservative differences in "moral" intuitions?". Social Justice Research 27: 413-431. DOI: http:// dx.doi.org/10.1007/s11211-014-0223-5

Kumashiro, K. K. 2000. "Toward a theory of anti-oppressive education". Review of Educational Research 70: 25-53. DOI: http://dx.doi.org/10.3102/00346543070001025

Lerner, M. y D. Miller. 1978. "Just world research and the attribution process: looking back and ahead". Psycological Bulletin 85: 1030-1051. DOI: http://dx.doi. org/10.1037/0033-2909.85.5.1030

$\mathrm{Li}, \mathrm{Ch}$. 2016. "Confirmatory factor analysis with ordinal data: Comparing robust maximum likelihood and diagonally weighted least squares". Behavior Research Methods 48: 936-949. DOI: https://dx.doi.org/10.3758/s13428015-0619-7

Lipkus, I. 1991. "The construction and preliminary validation of a global belief in a just world scale and the exploratory analysis of the multidimensional belief in a just world scale". Personality Individual Differences 12: 1171-1178. DOI: http://dx.doi.org/10.1016/0191-8869(91)90081-L

Lledó, A., M. Nieto, D. Lloret y B. Aldeguer. 2010. “Una aproximación al estudio de la identificación partidista e ideología política a través de la edad“. Psicología Política 41: 43-60.

Luhman, N. 2005. Confianza. Barcelona: Anthropos Editorial.

Maes, J. 1998. "Immanent justice and ultimate justice: two ways of believing in justice". Pp. 43-53 en Responses to victimizations and belief in the just world, editado por L. Montada y M. Lerner. New York: Plenum. DOI: http:// dx.doi.org/10.1007/978-1-4757-6418-5_2

Manzano-Arrondo, V. 2012. La Universidad Comprometida. Vitoria: Hegoa.

Manzano-Arrondo, V. 2014. "Il Barómetro Cittadino. La risposta dell'Universitá per unire didattica, ricerca, azione". Rivista Internazionale di EDAFORUM, 23: 1-22. 
Manzano-Arrondo, V. 2015. "Academia, evaluación y poder". RASE, 8: 197-222.

McCombs, M. 1996. "Influencia de las noticias sobre nuestras imágenes del mundo". Pp. 13-34 en Los efectos de los medios de comunicación. Investigaciones y teorías, editado por J. Bryant y D. Zillmann. Barcelona: Paidós.

Mirisola, A., M. Roccato, S. Russo, G. Spagna y A. Vieno 2014. "Societal threat to safety, compensatory control, and right-wing authoritarianism". Political Psychology 35: 795-812. DOI: http://dx.doi.org/10.1111/pops.12048

Morales, P. 2000. Medición de actitudes en psicología y educación. Madrid: Universidad Pontificia de Comillas.

Morales, P., B. Urosa y Á. Blanco. 2003. Construcción de escalas de actitudes tipo Likert. Madrid: La Muralla

Nafstad, H. E., R. M. Blakar, E. Carlquist, J. M. Phelps y K. Rand-Hendriksen. 2007. "Ideology and power. The influence of current neo-liberalism in society". Journal of Community y Applied Social Psychology 17: 313-327. DOI: http://dx.doi.org/10.1002/casp.931

Orjuela, L. J. 2007. "Ideologías, tecnocracia y sociedad: implicaciones para América Latina". Pp. 219-234 en Filosofía y teorías políticas entre la crítica y la utopía editado por G. Hoyos. Buenos Aires: CLACSO.

Pérez, E. R. y L. Medrano. 2010. "Análisis factorial exploratorio: bases conceptuales y metodológicas". Revista Argentina de Ciencias del Comportamiento 2: 58-66.

Petit, L. y G. L. Costa. 2011. "Dominancia social: el género como jerarquía social. Hologramática 3: 71-83.

Prat, R. y Doval, E. 2003. "Construcción y análisis de escalas". Pp. 43-89 en Análisis multivariante para las Ciencias Sociales, editado por J. Lévy, y J. Varela. Madrid: Prentice-Hall.

Pratto, F., J. Sidanius, J., Stallworth, L. M. y Malle, B. F. 1994. "Social dominance orientation: a personality variable predicting social and political attitudes". Journal of Personality and Social Psychology 67: 741-763. DOI http://dx.doi.org/10.1037/0022-3514.67.4.741

Reicher, St. 2004. "The context of social identity: domination, resistance, and change". Political Psychology 25: 921-945. DOI: http://dx.doi.org/10.1111/j.14679221.2004.00403.x
Rodrigo, M. y P. Medina. 2006. "Posmodernidad y crisis de identidad". Revista Científica de Comunicación e Información 3: 125-146.

Rodríguez, G. y E. Castañeda. 2006. "Contenidos estereotípicos, preferencia y actividad política“. Psicología Política, 33, 63-74.

Rottenbacher, J. M. y M. Schmitz. 2012. "Conservadurismo político y tolerancia hacia comportamientos transgresores“. Psicología Política 44: 34-56.

Ryan, R. M. y K. W. Brown. 2003. "Why we don't need selfesteem: On fundamental needs, contingent love, and mindfulness". Psychological Inquiry 14: 71-82.

Saavedra, J. 2007. "Adquirir la identidad en una comunidad de objetos: la identidad social dentro de la sociedad de consumo". Nómadas. Revista crítica de Ciencias Sociales y Jurídicas 16: 1-18.

Scurtu, M. Cl., V. Manzano-Arrondo y J. Fr. Rodríguez. 2016. "Selección de palabras de contenido referencial. Una propuesta metodológica“. Psicológica 37: 151-168.

Sibley, Chr. G. y J. Duckitt. 2010. "The ideological legitimation of the status quo: longitudinal tests of a social dominance model“". Political Psychology 31: 109-137. DOI: http://dx.doi.org/10.1111/j.1467-9221.2009.00747.x

Sidanius, J., E. Devereux y F. Pratto. 1992. "A comparison of symbolic racism theory and social dominance theory as explanations for racial policy attitudes". The Journal of Social Psychology 132: 377-395. DOI: http://dx.doi. org/10.1080/00224545.1992.9924713

Sidanius, J., F. Pratto, M. y M. Mitchell. 2004. "In-group identification, social dominance orientation, and differential intergroup social allocation". The Journal of Social Psychology 13: 151-167. DOI: http://dx.doi.org/10.108 0/00224545.1994.9711378

Swann, W. B. y M. D. Buhrmester. 2015. "Identity fusion". Current Directions in Psychological Science 24: 52-57. DOI: http://dx.doi.org/10.1177/0963721414551363

Vázquez, C. 2004. "Refortalecimiento: un debate con el empowerment". Revista Interamericana de Psicología 38: 41-51.

Zakrison, I. 2005. "Construction of a short version of the RightWing Authoritarianism (RWA) scale". Personality and Individual Differences 39: 863-872. DOI: http://dx.doi. org/10.1016/j.paid.2005.02.026

VICENTE MANZANO-ARRONDO. Licenciado en Filosofía y Ciencias de la Educación. Doctor en Psicología. Doctor en Pedagogía. Doctor en Economía. Fue presidente de SIPIE y director de la revista Metodología de Encuestas. Premio al mejor profesor de psicología por la Asociación Estatal de Estudiantes de Psicología. Miembro fundador de Universidad y Compromiso Social; Comunidad de Investigación, Acción y Desarrollo para superar la Opresión; y Coalición para el Estudio de la Salud, el Poder y la Diversidad. 


\section{ApÉndice 1.}

\section{Enunciados eliminados tras la fase de consulta a expertos}

1. Cada cual construye su propia ideología.

2. El saber técnico es suficiente para gobernar.

3. Gobernar consiste en poner en práctica una forma de ver las cosas.

4. Hay ideologías buenas e ideologías dañinas.

5. Hay ideologías que matan.

6. Las cosas son como son.

7. Las decisiones tomadas por razonamientos lógicos son mejores que las tomadas por opciones ideológicas.

8. Las ideologías extremas son negativas.

9. Las ideologías las crean los líderes.

10. Las ideologías llevan al desprecio.

11. Las mejores soluciones son las que se toman atendiendo solo a cómo son realmente las cosas.

12. Me gusta coincidir con personas que sigan mi propia ideología.

13. Mi forma de ver y estar en el mundo no sigue ninguna ideología.
14. Ni comparto ni deseo compartir ninguna ideología.

15. No creo en ninguna ideología.

16. No es más peligroso el que tiene una ideología distinta, sino el que no tiene ninguna.

17. No hay ideologías buenas o malas, sino solo distintas.

18. No hay mejor ideología que comer cada día.

19. Para seguir una ideología tienes que anularte como persona.

20. Pienso que las personas que describen las cosas de un modo distinto a como yo lo hago están equivocadas.

21. Prefiero a las personas que ven el mundo como yo lo veo.

22. Procuro juntarme con personas que tienen la misma ideología que yo.

23. Respeto que haya gente con ideologías, pero no que decidan por ello.

24. Todas las ideologías son igualmente respetables.

25. Una ideología es idolatrar algo o a alguien.

26. Una ideología se mete en la cabeza y no te deja pensar.

27. Ya no se lleva tener una ideología. 